\title{
COEFFICIENTE DI DIFFUSIONE DI ELETTRONI IN UN GAS
}

\author{
Donato Palumbo
}

$\grave{E}$ noto come il calcolo rigoroso, anche sotto ipotesi molto restrittive, del coefficiente di diffusione di elettroni lenti in un gas, presenti delle difficoltà quando si voglia tener conto della persistenza della velocità nelle collisioni con la molecola gassosa $\left({ }^{1}\right)\left({ }^{2}\right)$.

Di solito si tiene conto di questo fenomeno sostituendo al cammino libero medio un cammino allungato, mediante un opportuno coefficiente.

Vogliamo dimostrare come a questo risultato si possa pervenire generalizzando un ragionamento di Townsend $\left({ }^{3}\right)$, da questo Autore limitato al caso in cui la direzione della velocità dell'elettrone dopo la collisione possa ritenersi indipendente dalla direzione iniziale della velocità, il che non sempre può assumersi rero, come mostra la teoria del fenomeno.

1. - Cominciamo col dimostrare come il coefficiente di diffusione possa mettersi in relazione alla variazione del raggio d'inerzia di una distribuzione di elettroni provenienti da un punto $O$.

L'equazione generale della diffusione è

$$
\boldsymbol{K} \nabla^{\mathbf{2}} \varphi=\frac{\partial \varphi}{\partial t}
$$

Se si hanno $N$ elettroni e se indichiamo con $\varphi d \tau$ il numero di elettroni presenti dopo un tempo $t$ nell'elemento di volume $d \tau$, per il quadrato del raggio d'inerzia si ha:

$$
Q^{2}=\frac{1}{N} \int \varphi r^{2} d \tau
$$

dove l'integrale è esteso a tutto lo spazio, da cui

$$
\frac{d\left(\varrho^{2}\right)}{d t}=\frac{1}{N} \int \frac{\partial \varphi}{\partial t} r^{2} d \tau=\frac{K}{N} \int \nabla^{2} \varphi \cdot r^{\circ} d \tau
$$

Essendo il fenomeno a simmetria sferica attorno ad $O$ questa può scriversi 


$$
\frac{d\left(\varrho^{2}\right)}{d t}=\frac{4 \pi K}{N} \int_{0}^{\infty} \frac{\partial}{\partial r}\left(r^{2} \frac{\partial \varphi}{\partial r}\right) r^{2} d r
$$

ed integrando due volte per parti:

$$
\frac{d\left(\mathrm{Q}^{2}\right)}{d t}=\frac{4 \pi K}{N}\left[\frac{\partial \varphi}{\partial r} r^{4}\right]_{0}^{\infty}-\frac{8 \pi K}{N}\left[r^{3} \varphi\right]_{0}^{\infty}+\frac{24 \pi K}{N} \int_{0}^{\infty} r^{2} \omega d r
$$

I termini ai limiti si annullano, poiché $\varphi$ decresce esponenzialmente (") al crescere di $r^{2}$, e quindi:

$$
\frac{d\left(0^{2}\right)}{d t}=\frac{24 \pi K}{N} \int_{0}^{\infty} r^{2} \varphi d r=\frac{6 K}{N} \int p d \tau=6 K
$$

Poiché il fenomeno, considerato nel suo meccanismo, è discontinuo, conviene scrivere la $[1]$, dopo integrazione:

$$
K=\frac{1}{6} \frac{\Delta\left(\rho^{i}\right)}{t}
$$

essendo $\Delta\left(\varrho^{2}\right)$ la variazione del quadrato del raggio d'inerzia nel tempo $t$, tempo che puó assumersi abluastanza piccolo rispetto alla lentezza dei fenomeni di diffusione, ma in modo che in esso ciascun eleftrone subisca un gran numero di urti.

2. - Nell'ipotesi che tutti gli urti siano elastici; trascurando la massa dell'elettrone rispetto a quella delle molecole del gas (sicché l'elettrone non perda energia nelle collisioni); clse dopo ciascun urto la direzione delle velocità dell'elettrone sia indipendente da quella iniziale, Townsend trova

$$
K=\frac{l U}{3}
$$

essendo $l$ il cammino libero medio degli elettroni ed $U$ la loro velocità. Vogliamo generalizzare questo procedimento al caso in cui non possa farsi l'ultima ipotesi dichiarata. Supponiamo che un elettrone dopo la collisione con una molecola abljia una probabilità $f(\vartheta) d \Omega$ di muoversi in una direzione interna all'angolo solido $d \Omega$ compreso tra due coni di apertura $\hat{v} \vartheta+d \vartheta$, aventi per asse la direzione della velocità dell'elettrone prima dell'urto. Deve essere: 


$$
\int_{i} f(\vartheta) d \Omega=1
$$

essendo $S$ la sferia unitaria col centro nel punto di collisione.

Poniamo ancora $\int f(4) \cos \vartheta d \Omega=\alpha$. Evillentemente si avrà $\alpha=O$ nella ipotesi che dopo l'urto le direzioni della velocità siano tutte equalmente probabili. Consideriamo un elettrone uscente da $O$ con velocità $U$. Il primo urto avverrà in una sfera $S$, di rauggio $l_{1}$, ed in tale istante il quadrato del raggio d'inerzia sarà $\varrho_{1}{ }^{2}=l_{1}{ }^{2}$. Il secondo urto avverrà su una certa sfera $S_{2}$ di raggio $L_{\text {.", }}$ avente il centro in un punto $P_{1}$ di $S_{1}$. Ma non tutti gli clementi di superficie

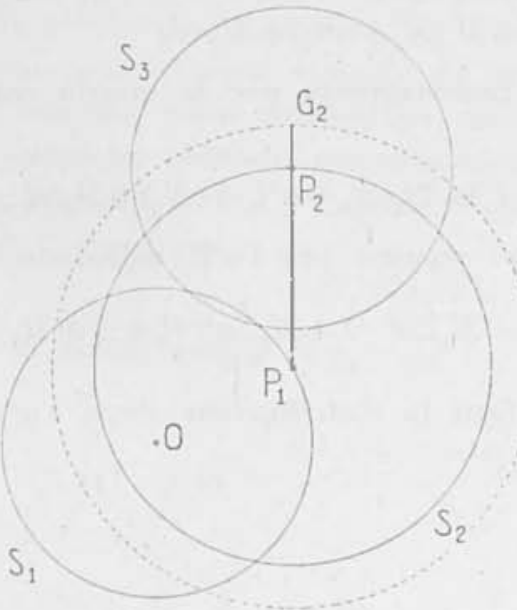

Fig. 1

fferica intercetlati dall'angolo solido $d \Omega$, sono ugualmente probabili, ma a ciascuno spetta un coefficiente $f(y) d \Omega$.

Ne viene che il centro di massa della probabilità di presenza dell'elettrone si trova in un punto $G$, sul prolungamento di $O P_{1}$, e

$$
P_{1} G_{1}=l_{\mathrm{s}} \frac{\int^{\mathrm{s}}(\mathrm{y}) \cos \vartheta d \Omega}{f(\vartheta) d \Omega}=l_{\mathrm{n}} c
$$

Il quadrato del raggio d'inerzia di questa distribuzione rispetto a $P_{1}$ è $l_{i}$, rispetto a $G_{1} \ldots-\alpha^{\cdot} l_{2}^{2}$ e quindi rispetto ad $O$

$$
Q_{2}^{2}=l_{2}^{2}-\alpha^{2} l_{2}^{2}+\left(l_{1}+\alpha l_{2}\right)^{2}=l_{1}^{2}+l_{z}^{2}+2 \alpha l_{1} l_{2}
$$


Il terzo urto avverrà su una sfera $S_{3}$ di raggio $l_{3}$ ed avente il centro su un certo punto $P_{\text {g }}$ di una delle sfere $S_{\text {s. }}$. In tale istante il quadrato del raggio di inerzia rispetto a $P_{3}$ sarà $l_{3}^{2}$, ed il centro di massa su un punto $G_{\text {o }}$ situato sul prolungamento di $P_{1} P_{\text {., a }}$ a distanza $a l_{3}$ da $P_{3}$, quindi il quadrato del raggio d'inerzia rispetto a $P$., e

$$
l_{0}{ }^{2}+l_{3}{ }^{2}+2 \alpha l_{n} l_{3}
$$

mentre il centro di massa relativo alla collisione in $P_{1}$ è sul prolungramento di $O P_{1}$ a distanza $a l-f-a l$ ) da $P_{\text {. }}$.

Ne viene per il quadrato del raggio d'inerzia rispetto ad $O$ al momento della terza collisione:

$$
\begin{aligned}
& \varrho_{3}^{2}=l_{3}^{2}-\alpha^{2} l_{3}{ }^{2}+\left(l_{9}+\alpha l_{3}\right)^{2}-\alpha^{2}\left(l^{2}+\alpha l_{3}\right)+\left(l_{1}+\alpha l_{9}+\alpha^{2} l_{3}\right)^{2}= \\
& =l_{1}^{2}+l_{2}{ }^{2}+l_{3}{ }^{2}+2 l_{1}\left(\alpha l_{2}+\alpha^{2} l_{3}\right)+2 l_{2} \cdot \alpha l_{3}
\end{aligned}
$$

Ripetendo lo stesso ragionamento per la quarta collisione si troverelble:

$$
\varrho_{4}{ }^{2}=l_{1}{ }^{2}+l_{2}{ }^{2}+l_{3}{ }^{2}+l_{4}{ }^{2}+2 l_{1}\left(\alpha l_{2}+\alpha^{2} l_{3}+\alpha^{3} l_{4}\right)+2 l_{2}\left(\alpha l_{3}+\alpha^{2} l_{4}\right)+2 l_{3} \cdot \alpha l_{4}
$$

Lo stesso metorlo può seguirsi per l'n ${ }^{\text {ma }}$ collisione e si trova:

$$
\varrho_{\mathbf{n}}{ }^{2}=\sum_{\mathbf{i}=1}^{n} l_{i} i+2 l_{1} \sum_{i=2}^{n} \alpha^{i-1} l_{i}+2 l_{2} \sum_{i=3}^{n} \alpha^{i-i} l_{i}+\cdots+2 l_{n-1} \alpha l_{n}
$$

Consideriamo infatti la distribuzione dopo l'n $n^{\mathrm{ma}}$ collisione, pro-

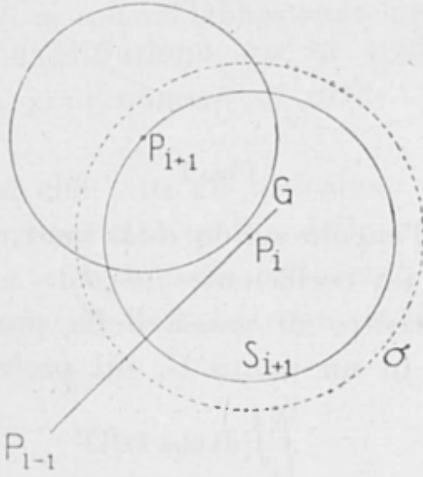

Fig. 2

veniente dalla $i^{\text {ma }}$ collisione in $P_{i}$. I centri di massa si trovano con la consueta distribuzione angolare intorno alla $P_{\mathrm{i}-1} P_{\mathrm{i}}$, su una sfera $\sigma$ di raggio $l_{\mathrm{i}+1}+\delta_{i+1}$ e di centro $F_{\mathrm{i}}$ : il centro di massa della distribuzione proveniente da $P_{1}$ è perciò sulla $P_{i-1} P$, a distanza $\alpha\left(l_{i+1}+\delta_{+1}\right)$ da $P_{\mathrm{i}}$. Quindi per passare dal valore del quadrato del raggio d'inerzia 
rispetto a $P_{\mathrm{i}}$ a quello rispetto a $P_{:-1}$, hisogna sottrarre $\alpha^{2}\left(l_{\mathrm{i}+1}+\delta_{\mathrm{i}+1}\right)=$ ed aggiungere $\left(l_{i}+\alpha l_{i+1}+\alpha \delta_{i+1}\right)^{2}$

cioè aggiungere

$$
l_{i}^{2}+2 l_{i}\left(\alpha l_{i+1}+\alpha \delta_{i+1}\right)
$$

Da quanto detto risulta ancora che $\delta_{i}=\alpha\left(l_{i+1}+\delta_{i+1}\right)$; e, poiché $\delta_{n-1}$ ovviamente è uguale ad $\alpha l_{n}$, si possono trovare per ricorrenza tutti i $\delta_{i}$. Sostituendoli nella [5] e sommando si trova la [4].

3. - La probabilità che l'mo cammino albbia un valore compreso tra $l_{\mathrm{i}}$ ed $l_{\mathrm{i}}+d l_{\mathrm{i}}$ è proporzionale a $e^{-\beta l_{\mathrm{i}}} d l_{\mathrm{i}}$, con $\frac{l}{\beta}=l$, cammino libero medio degli elettroni di velocità $U$. Potendosi supporre che il valore di un certo cammino non dipenda da quello dei precedenti, può applicarsi il teorema delle probabilità composte, e quindi la probabilità che i cammini abbiano successivamente valori compresi tra $l_{1}, l_{1}-d l_{1} ; l ., l_{.}+d l_{l} ; \ldots ; l_{\mathrm{n}}, l_{\mathrm{n}}+d l_{\mathrm{n}}$, è proporzionale a

$$
e^{-\beta\left(l_{1}+l_{2}+\cdots+l_{n}\right)} d l_{1} d l_{2} \ldots d l_{n}
$$

Ne viene per il valore medio di $\varrho_{n}^{2}$ :

$$
\begin{aligned}
& \overline{\varrho_{n}}=\frac{1}{A} \int_{0}^{\infty} \cdots \int_{0}^{\infty} \varrho_{n}^{2} e^{-\hat{\mu}\left(i_{1}+i_{2}+\cdots+i_{n}\right)} d l_{1} \ldots d l_{n} . \\
& \operatorname{con} A=\quad \int_{0}^{\infty} \cdots \int_{0}^{\infty} e^{-\beta\left(l_{1}+L^{2}+\cdots+l_{n}\right)} d l_{1} \ldots d l_{\mathrm{n}}
\end{aligned}
$$

Eseguendo i calcoli, che si riducono alla ricerca del valor medio di termini del tipo $l_{i}^{2}$ ed $l_{i} i_{i}$, si trova:

$$
\overline{\varrho_{n}^{2}}=2 \iota^{2}\left(n+\sum_{i=2}^{n} \alpha^{i-1}+\sum_{i=3}^{n} \alpha^{i-2}+\cdots+\alpha\right)=2 l^{\xi}\left[\frac{n}{1-\alpha}-\alpha \frac{1-\alpha^{n}}{(1-\alpha)^{2}}\right]
$$

Il tempo $t$ trascorso è, a meno di piccole fluttuazioni ("),$\frac{n l}{U}$; poiché $\Delta\left(\varrho^{2}\right)=\overline{Q_{n}^{2}}$ per la $[2]$, e per valori di $n$ sufficientemente grandi, si ha:

$$
K=\frac{U}{3} \frac{l}{1-\alpha}
$$


formula che coincide con quella data dagli Autori citati, e che si riduce alla $\lceil 3\rceil$ per $\alpha=O$.

$\mathrm{Ci}$ è grato ringraziare l'on. prof. Enrico Medi per l'interessamento dimostratoci.

Palermo - Istituto di Fisica dell'Università - Dicembre 1949.

\section{RIASSUNTO}

Si generalizza un procedimento di Townsend per il calcolo del coefficiente di diffusione di elettroni in un gas, per il caso in cui la direzione della velocità dell'elettrone dopo ciascun urto non possa ritenersi indipendente dalla direzione della velocità prima dell'urto. $\mathrm{Si}$ perviene cosi alla nota espressione del cammino libero medio allungato.

\section{BIBL.IOGRAFIA}

(1) Bonel: Mécanique Statistique Classique, Cap. VII; Paris, 1925.

(2) Jeans: Theorie Dynamique des Gaz, Capp. X; XIII; Paris, 1925.

(3) Townseno: Electrons in Gases, Cap. I; London, 1947.

(1) Persico: Introduzione alla Fisica Matematica, Cap. VII; Bologna, 1945.

(7) Bones: Mécanique Statistique Classique, Cap. VII; p. 99; Paris, 1925. 